\title{
Stratified prokaryote network in the oxic-anoxic transition of a deep-sea halocline
}

\author{
Daniele Daffonchio ${ }^{1}$, Sara Borin ${ }^{1}$, Tullio Brusa ${ }^{1}$, Lorenzo Brusetti ${ }^{1}$, Paul W. J. J. van der Wielen ${ }^{2}$, Henk Bolhuis ${ }^{2}$, \\ Michail M. Yakimovv', Giuseppe D'Auria ${ }^{3}$, Laura Giuliano ${ }^{3}$, Danielle Marty ${ }^{4}$, Christian Tamburini ${ }^{4}$, \\ Terry J. McGenity ${ }^{5}$, John E. Hallsworth ${ }^{5}$, Andrea M. Sass ${ }^{5}$, Kenneth N. Timmis ${ }^{5,6}$, Anastasios Tselepides ${ }^{7}$, \\ Gert J. de Lange ${ }^{8}$, Andreas Hübner ${ }^{8}$, John Thomson ${ }^{9}$, Soterios P. Varnavas ${ }^{10}$, Francesco Gasparoni ${ }^{11}$, \\ Hans W. Gerber ${ }^{12}$, Elisa Malinverno ${ }^{13}$, Cesare Corselli ${ }^{13} \&$ Biodeep Scientific Party ${ }^{\star}$
}

\begin{abstract}
The chemical composition of the Bannock basin has been studied in some detail ${ }^{1,2}$. We recently showed that unusual microbial populations, including a new division of Archaea (MSBL1) ${ }^{3}$, inhabit the NaCl-rich hypersaline brine. High salinities tend to reduce biodiversity ${ }^{4}$, but when brines come into contact with fresher water the natural haloclines formed frequently contain gradients of other chemicals, including permutations of electron donors and acceptors, that may enhance microbial diversity, activity and biogeochemical cycling ${ }^{5,6}$. Here we report a $2.5-\mathrm{m}$ thick chemocline with a steep $\mathrm{NaCl}$ gradient at $3.3 \mathrm{~km}$ within the water column betweeen Bannock anoxic hypersaline brine ${ }^{7}$ and overlying sea water. The chemocline supports some of the most biomass-rich and active microbial communities in the deep sea, dominated by Bacteria rather than Archaea, and including four major new divisions of Bacteria. Significantly higher metabolic activities were measured in the chemocline than in the overlying sea water and underlying brine; functional analyses indicate that a range of biological processes is likely to occur in the chemocline. Many prokaryotic taxa, including the phylogenetically new groups, were confined to defined salinities, and collectively formed a diverse, sharply stratified, deep-sea ecosystem with sufficient biomass to potentially contribute to organic geological deposits.
\end{abstract}

High-precision sampling was conducted during cruises of the research vessel Urania equipped with the Modus-Scipack system (http://www.geo.unimib.it/BioDeep/Project.html; Fig. 1a). The vehicle Modus, connected by cable to the research vessel, held a second instrument, the Scipack, with a 10-m data transmission cable. The Scipack, consisting of a Rosette sampler equipped with a CTD (conductivity-temperature-depth probe) and a series of Niskin bottles, was connected to the Modus through the Sciskid, a module equipped with a pressure sensor for recording the pressure at which the Niskin bottles were closed (Fig. 1c). A camera on the Modus could provide an image of the Scipack entering the brine lake (Fig. 1b, and Supplementary Fig. S1). Immediately after sampling, the Modus-Scipack was raised, the Niskin bottles were retrieved and their contents were carefully fractionated on board ship by slowly recovering 0.5 -litre, 1-litre or 2-litre fractions from the bottom tap. These were then immediately analysed for salinity (Fig. 1d). The reconstructed interface salinity profile was strongly positively correlated $(r=0.98, P<0.001)$ with the CTD conductivity profile recorded in independent non-sampling casts (Fig. 2d), indicating that little or no mixing had occurred.

The interface halocline was about $2.5 \mathrm{~m}$ deep, in agreement with previous estimates that employed alternative sampling strategies ${ }^{1}$. Although biomass values fluctuated along the halocline, there were significantly greater numbers of microbial cells in the interface (about $10^{6}$ cells ml $^{-1}$ ) than in either the deep sea water or the underlying hypersaline brine, both of which had about $10^{4}$ cells $\mathrm{ml}^{-1}$ (Fig. 2a). Comparison of archaeal and total prokaryotic 16S ribosomal RNA gene abundance revealed that Bacteria and Archaea were present at equivalent levels in the oxic seawater above the hypersaline brine, as reported previously for the deep $\mathrm{sea}^{8}$. In contrast, the interface samples contained 20.5 and $1.8 \mathrm{pg} \mathrm{ml}^{-1}$ bacterial and archaeal 16S rRNA gene, respectively (Fig. 2b), contrary to the notion that extreme environments are dominated by Archaea but consistent with the low level of Archaea-mediated methanogenesis measured in the upper $2 \mathrm{~m}$ of the halocline (Fig. 2c). The high concentration of bacteria in the interface indicates that it acts as a barrier to particulates that sink through the marine water column, leading to an accumulation of organic carbon, nutrients and reactive surfaces that support microbial growth ${ }^{9}$. In addition, methane originating in the hypersaline brine will rise and enter the interface, where it can be oxidized under more energetically favourable conditions.

Levels of ATP, an important indicator of metabolic activity ${ }^{10}$, were significantly higher in the interface than in the overlying sea water and the brine lake (Fig. 2b), as were extracellular aminopeptidase and alkaline phosphatase activities (Supplementary Table S1). At certain depths of the interface, sulphate reduction rates (SRRs) showed appreciably higher values than those of the deep oxic sea water ${ }^{11}$, but the highest SRRs were measured in the underlying anoxic brine (Fig. 2c).

Down the halocline, nitrate concentration decreased from 6.6 to $0.3 \mu \mathrm{M}$, ammonium increased from 5 to $3,450 \mu \mathrm{M}$ (refs 2,12 ), sulphate, the most abundant electron acceptor along the halocline, increased from 31 to $84 \mathrm{mM}$, manganese increased from 0.4 to $8.3 \mu \mathrm{M}$ and redox potential decreased from $210 \mathrm{mV}$ to less than zero, indicating a total depletion of oxygen in the lower part of the chemocline (Fig. 2e-g). Electron acceptors and donors potentially

${ }^{1}$ CoNISMa, Ulr Università degli Studi di Milano, DiSTAM, 20133 Milan, Italy. ${ }^{2}$ Department of Microbial Ecology, CEES, University of Groningen, 9751 NN Haren, The Netherlands. ${ }^{3}$ Istituto per I'Ambiente Marino Costiero, CNR, 98122 Messina, Italy. ${ }^{4}$ LMGEM, UMR 6117 CNRS Université de la Mediterranée, 13288 Marseille, France. ${ }^{5}$ Department of Biological Sciences, University of Essex, Colchester, Essex CO4 3SQ, UK. ${ }^{6}$ Division of Microbiology, GBF, 38122 Braunschweig, Germany. ${ }^{7}$ Institute of Marine Biology of Crete, 71003 Heraklion, Greece. ${ }^{8}$ Faculty of Geosciences, Geochemistry, Utrecht University, 3584 CD Utrecht, The Netherlands. ${ }^{9}$ Southampton Oceanography Centre, Southampton SO14 3EE, UK. ${ }^{10}$ Department of Geology, University of Patras, 26100 Patras, Greece. ${ }^{11}$ Tecnomare S.p.A., ENI Group, 30124 Venice, Italy. ${ }^{12}$ Technische Fachhochschule Berlin, University of Applied Science, 13353 Berlin, Germany. ${ }^{13}$ CoNISMa, Ulr Università degli Studi di Milano Bicocca, DGSG, 20126 Milan, Italy.

${ }^{\star} A$ list of participants and affiliations appear at the end of the paper. 
available for metabolic processes in the interface are thus variously distributed along the gradient. Whereas $\mathrm{Na}^{+}$showed chemically conservative behaviour along the halocline, dissolved manganese, nitrate, ammonium and sulphate concentrations exhibited nonlinear slopes, demonstrating non-conservative behaviour presumably reflecting biologically mediated redox cycling ${ }^{13}$ (Supplementary Fig. S2).

Detailed vertical profiles of biological parameters showed two maxima in diversity at about $8 \%$ and $15-22 \%$ salinity, which corresponded to maxima in biomass, determined by total cell counts and prokaryote $16 \mathrm{~S}$ rRNA gene abundance analysis $(r=0.93$, $P<0.001$; Fig. 2a), and in ATP concentration $(r=0.74, P<0.01$ and $r=0.64, P<0.05$; Fig. $2 b$ ). The decreases in these biological parameters in the $10-15 \%$ salinity range, and the low SRR in this region, can be explained by a depletion of nutrients and organic substrates from above and below, and/or a localized shortage of energetically favourable redox couplings (for example nitrate and oxygen are depleted at this point; Fig. 2e, f).

$\mathrm{NaCl}$ is well known for generating high osmotic potentials, reducing the mole fraction of water, and its kosmotropic (ordergenerating) activity stabilizes cellular macromolecules and stiffens membranes (Supplementary Fig. S3): such parameters influence the environmental windows of growth for any microbial species. In contrast with other hypersaline environments, such as coastal solar salt pans ${ }^{4}$, bacterial diversity in the Bannock interface was higher than that found in the adjacent low-salt habitat (the overlying deep seawater), as shown by Shannon-Weaver indices derived from amplified ribosomal intergenic spacer analysis (ARISA) of Bacteria at 18 different salinities (Fig. 2a). To identify the Bacteria present at different locations in the halocline, clone libraries of RT-PCR amplicons of $16 \mathrm{~S}$ rRNA (complementary DNA) and of PCR amplicons of 16S rRNA genes were generated; 539 clones, including 179 cDNA clones representing the more metabolically active microbial fraction, were sequenced. This analysis confirmed the high bacterial phylogenetic diversity suggested by the Shannon-Weaver indices and identified four new candidate divisions designated Mediterranean Sea Brine Lake groups 3-6 (MSBL3-6) and a dominant candidate division, newly named MSBL2 (Supplementary Fig. S4). Candidate division MSBL2 is phylogenetically related to the candidate division SB1, previously identified in the interface of the Shaban Deep in the Red Sea ${ }^{14}$. MSBL2-related sequences were also detected in the interfaces of other hypersaline basins, but were absent from the underlying brines or the overlying sea water ${ }^{3}$, which indicates that bacteria belonging to the MSBL2 division are specifically adapted to the seawater-hypersaline brine interfaces.

The deeper part of the halocline (22.2-25.0\% salinity) was inhabited by MSBL2, by Sphingobacteriaceae and by candidate divisions KB1 (refs 14, 15) and MSBL3-6 (Fig. 3a). The candidate division KB1 includes species related to Thermotogales that have been found in brine lakes and their sediments in the Red Sea ${ }^{14,15}$, and in other anaerobic hypersaline sediments ${ }^{16}$. Despite being less abundant than those of MSBL2, bacteria of MSBL3-6 candidate divisions were found only in the deeper part of the halocline, indicating that they are adapted to high salinity. Archaeal clones
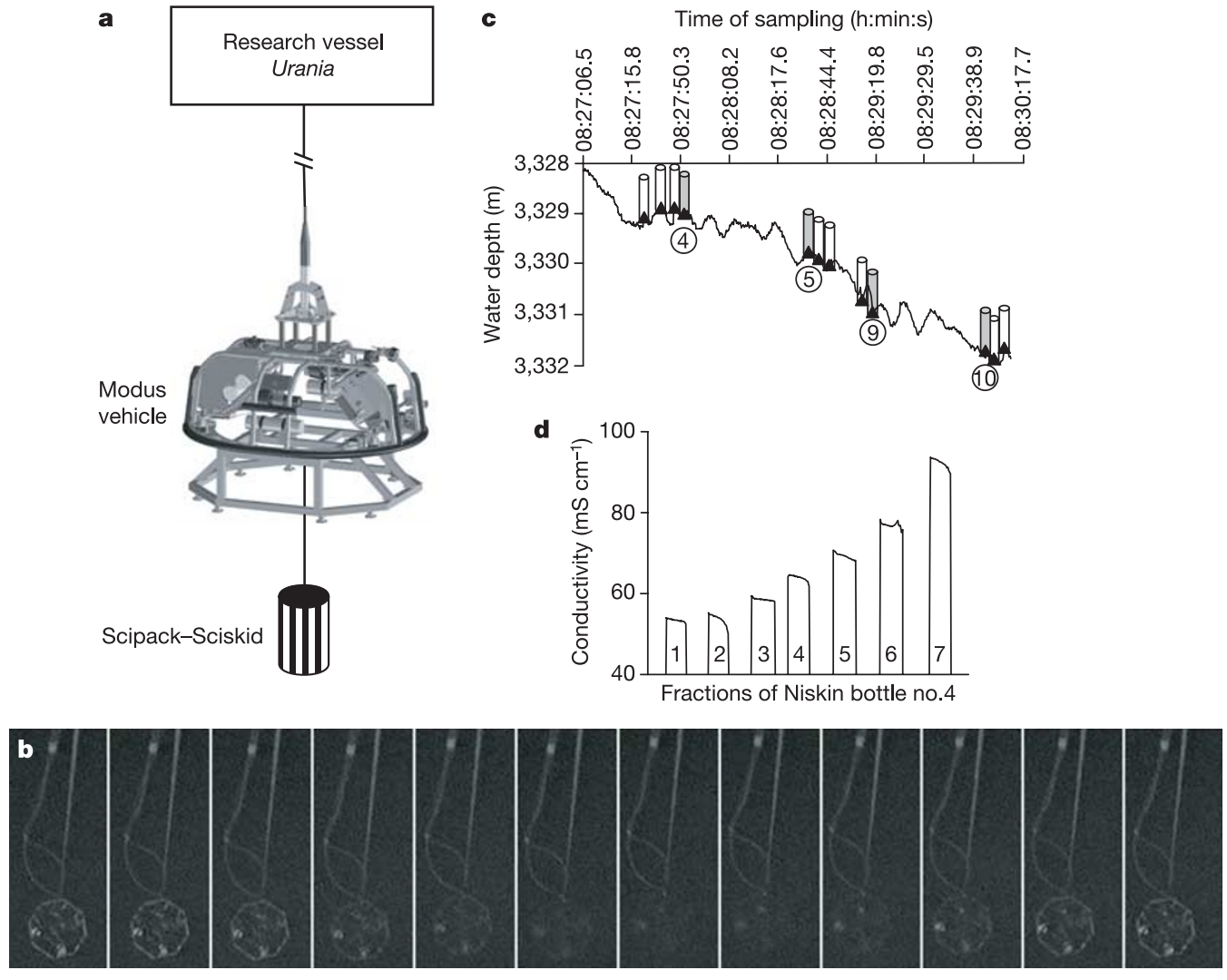

Figure 1 Procedures for sample recovery from the seawater-Bannock brine interface. a, Schematic drawing of the Modus-Scipack system illustrating the Modus vehicle holding the Scipack-Sciskid unit consisting of a Rosette sampler equipped with a CTD and a series of Niskin bottles (Scipack), and a pressure sensor (Sciskid) for recording the pressure at which the Niskin bottles were closed. b, Photographs of the Scipack Rosette sampler at 0.5-s intervals while going into and out of the seawater-Bannock brine interface obtained during a video survey from the Modus vehicle (from left to right). The disappearance of the Scipack is caused by the transmittance change resulting from the high salinity of the brine. c, This sampling system yielded the exact time and depth at which each Niskin bottle (represented by cylinders over the curve) was closed. In this way it was possible to determine at which point of the conductivity curve during the cast the bottles were closed. The circled numbers below selected bottles identify those Niskin bottles (shaded) that were subsampled on board ship for the characterization of the gradient. d, Example of conductivity measurements of the different water fractions sub-sampled from Niskin bottle no. 4 reported in c, using the conductivity probe from the Scipack. 


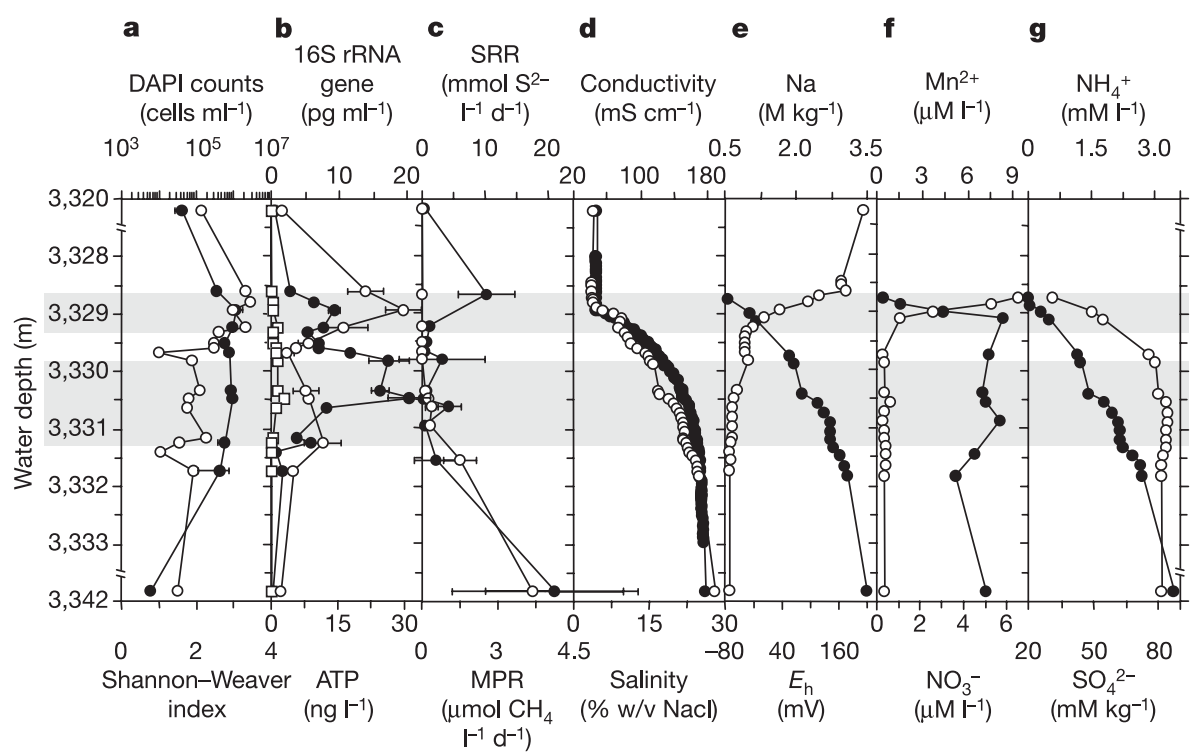

Figure 2 Microbiological and geochemical profiling of the seawaterBannock brine interface. a, Filled circles, DAPI microbial counts; open circles, Shannon-Weaver indices calculated from ARISA data. b, Filled circles, prokaryote $16 \mathrm{~S}$ rRNA gene abundance; open squares, Archaea $16 \mathrm{~S}$ rRNA gene abundance; open circles, ATP. c, Filled circles, SRR; open circles, methane production rate (MPR). d, Filled circles, conductivity; open circles, salinity. e, Filled circles, sodium; open circles, redox potential $\left(E_{\mathrm{h}}\right)$. f, Filled circles, dissolved manganese; open circles, nitrate. g, Filled circles,
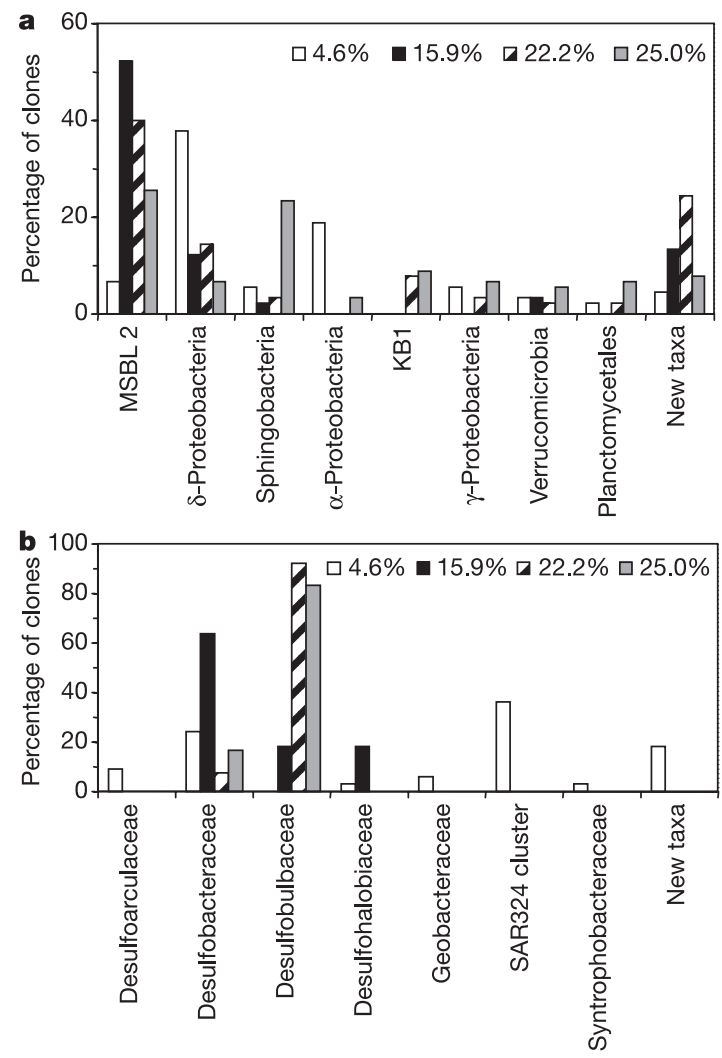

Figure $3 \mid$ Distribution of prokaryotic diversity at different salinities along the seawater-Bannock brine interface. a, Percentage distribution of $16 \mathrm{~S}$ rRNA gene clones belonging to different bacterial phylogenetic groups. New divisions MSBL3 -6 are included under 'new taxa'. b, Percentage distribution of different families of the $\delta$-Proteobacteria. ammonium; open circles, sulphate. Shading indicates zones with relatively high biological activity. Conductivity data in c were obtained during a non-sampling cast by continuous measurements with a conductivity probe mounted on the Scipack system. All the data were from station AB27SCI except values for deep sea water and Bannock brine that were from station AB29SCI. Error bars indicate standard deviations. For sodium, dissolved manganese, nitrate, ammonium and sulphate, standard deviations were $2-5 \%$ of each value.

related to members of candidate divisions MSBL1, previously found to dominate in the brine ${ }^{3}$, and ANME-1, associated with anaerobic oxidation of methane ${ }^{17}$, were found in cDNA libraries generated from samples from the deeper part of the halocline, namely the anoxic zone where methane and sulphate coexist. Sulphate-reducing $\delta$-Proteobacteria were abundant, comprising $6-15 \%$ of the clones in the libraries from all anoxic levels of the interface. Desulfobacteriaceae and Desulfobulbaceae were found mainly at lower and higher salinities, respectively (Fig. 3b), indicating specific adaptation of these two groups to the different prevailing environmental factors of salinity, redox potential, oxygen ${ }^{18,19}$, sulphate concentration and availability of organic substrates.

Eighty-four bacterial isolates were cultured from the Bannock interface; on the basis of partial 16S rRNA sequences they were assigned to the Firmicutes, Bacteroidetes, $\alpha$-Proteobacteria, $\gamma$-Proteobacteria and $\varepsilon$-Proteobacteria (Supplementary Table S2). The isolates belonged to divisions widely represented in the rRNA libraries; however, they did not correspond to any specific sequence found in the libraries (Supplementary Fig. S4). Most isolates were moderately halophilic facultative anaerobes, able to grow aerobically or by fermentation or denitrification, and therefore well adapted to the seawater-brine interface. In particular, a Halothiobacillus species was able to aerobically oxidize thiosulphate, with $\mathrm{CO}_{2}$ as sole carbon source, over a $\mathrm{NaCl}$ range of $0.5-23 \%$. In addition, two obligately anaerobic, fermentative, halophilic isolates had 99.8\% 16S rRNA sequence similarity to Halanaerobium sp. KT-2/3-3, which was isolated from a similar seawater-brine interface in the Kebrit deep ${ }^{20}$.

Two obligately anaerobic strains, one from the Bacteroidetes and one from the $\varepsilon$-Proteobacteria from the same enrichment, had less than $92 \% 16$ S rRNA sequence similarity to known organisms. Both were moderate halophiles, growing between $2 \%$ and $12 \% \mathrm{NaCl}$ and between $0.1 \%$ and $9.0 \% \mathrm{NaCl}$, respectively. The Bacteroidetes isolate fermented a variety of sugars and biopolymers as sole sources of carbon and energy, whereas the $\varepsilon$-Proteobacteria member fermented a variety of organic acids, and used nitrate, nitrite, thiosulphate, trimethylamine $\mathrm{N}$-oxide, dimethylsulphoxide and elemental sulphur as terminal electron acceptors with formate and acetate as electron 
donors. It can therefore be envisaged that these organisms, which are highly suited to life in Bannock interface, would benefit mutually from the degradation of organic polymers, which are likely to accumulate at the interface ${ }^{9}$, coupled to the respiration of sulphur compounds or nitrate.

Sulphate reducers, despite being abundantly represented in clone libraries, escaped cultivation. Nevertheless, analysis of our isolates confirmed that a range of biological processes-such as fermentation and the oxidation of reduced sulphur species and organic matter coupled to a variety of terminal electron acceptors-are likely to occur in the interface.

In conclusion, the Bannock brine lake-seawater interface is revealed here to be a deep-sea microbial oasis, consisting of a biomass-dense, metabolically active microbial ecosystem that receives methane from the hypersaline brine below and organic particulates sedimenting through the water column from above, recycles this organic matter and interconverts diverse ionic species, which are used and reused as electron acceptors and donors. Although functionally analogous to the deep-sea floor, which also collects and recycles sinking particulates, the interfaces of deep hypersaline basin lakes differ significantly in the stress imposed by their steep halocline, and consequently have selected for stratified microbial communities containing new organisms, including divisions of bacteria not previously described. Recent work detailing the retrieval of novel enzymes from the analogous Urania basin interface ${ }^{21}$ would suggest that this new phylogenetic diversity reflects new functional activity. Such biomass-rich environments are stable over hundreds of years, constituting localized sources of organic matter that may contribute to the geological record, for example in the form of sapropels ${ }^{22,23}$.

\section{METHODS}

Sampling of seawater-brine interface in Bannock basin. Sampling of the Bannock basin was conducted from the research vessel Urania at location $34^{\circ} 21.640^{\prime} \mathrm{N}, 20^{\circ} 02.260^{\prime} \mathrm{E}$ in 2001 , location $34^{\circ} 17.949^{\prime} \mathrm{N}, 20^{\circ} 00.985^{\prime} \mathrm{E}$ (station BD29CT) in 2002, and at locations $34^{\circ} 17.488^{\prime} \mathrm{N}, 20^{\circ} 00.692^{\prime} \mathrm{E}$ (AB27SCI) and $34^{\circ} 17.397^{\prime} \mathrm{N}, 20^{\circ} 00.709^{\prime} \mathrm{E}$ (AB29SCI) in 2003. The halocline water fractions, sampled with the Modus-Scipack system, were analysed as reported below for microbial activity, microbial abundance and diversity, physicochemical properties, and isolation of microbial strains.

Activity measurements. Aminopeptidase and phosphatase activities were determined with the fluorogenic substrates L-leucine-7-amino-4-methylcoumarin and 4-methylumbelliferyl phosphate with the use of the multiconcentration kinetic method at concentrations ranging from 0.05 to $10 \mu \mathrm{M}$ (ref. 24). Methane production rates were determined by measuring the production of methane with a gas chromatograph equipped with a flame ionization detector ${ }^{3}$. Sulphate reduction rates were determined by measuring the $\left[{ }^{35} \mathrm{~S}\right]$ sulphide production from radiolabelled sulphate $(1-2 \mu \mathrm{Ci}$ $\left[{ }^{35} \mathrm{~S}\right]$ sulphate) with standard methods. ATP was measured on triplicate $10-\mathrm{ml}$ samples filtered through $0.22-\mu \mathrm{m}$ pore-size filters. ATP was extracted and measured directly on the filter with the luciferin-luciferase-based biomass test kit (Promicol), with a luminometer, following the instructions of the manufacturer. Relative luminescence units were converted to ATP concentrations with the use of a standard ATP curve.

DNA/RNA-based analysis and direct cell count. Samples were filtered onto sterile $0.22-\mu \mathrm{m}$ pore-size filters that were stored at $-20^{\circ} \mathrm{C}$ in $2 \mathrm{ml}$ of sterile lysis buffer (EDTA $40 \mathrm{mM}$, Tris- $\mathrm{HCl} 50 \mathrm{mM} \mathrm{pH} 5.8$, sucrose $0.75 \mathrm{M}$ ). DNA was extracted from each filter as described previously ${ }^{3}$. Extracted DNA was quantified by comparative agarose-gel electrophoresis with known quantities of lambda phage DNA. 4,6-Diamidino-2-phenylindole (DAPI) staining was performed as described previously ${ }^{3}$. Real-time PCR experiments for the quantification of total Prokaryotes and Archaea were performed by Taqman assays as described elsewhere ${ }^{25}$.

From each DNA fraction along the salinity gradient, ARISAs were performed in accordance with an established protocol ${ }^{26}$. As a parameter for the structural diversity of a bacterial community a Shannon-Weaver $\operatorname{index}^{27}(H)$ was calculated for representative ARISA profiles by using the function $H=-\Sigma\left(n_{i} / N\right) \ln \left(n_{i} / N\right)$, where $n_{i}$ is the height of a peak and $N$ is the sum of all peak heights in an ARISA profile. The construction and sequencing of $16 \mathrm{~S}$ rRNA gene libraries from fractions with different salinities were performed with a previously described procedure ${ }^{3}$. Sequences were aligned with the use of ARB software $^{28}$, and operational taxonomic unit (OTU) distribution at different salinities was calculated. Good coverage of the dominant OTU population was confirmed with rarefaction analysis of the clone libraries. Sequences of 16S rRNA genes showing more than $97 \%$ homology were considered to belong to the same OTU. For RNA extraction and reverse transcription the RNA/DNA Mini Isolation kit (Qiagen) and Superscript II reverse transcriptase (Invitrogen) were used. Geochemical analyses and salinity-related measurements. The redox potential in each halocline fraction was measured on board ship with a portable $E_{\mathrm{h}}$ meter $^{29}$. Major elements in brines were analysed by inductively coupled plasma atomic emission spectrometry at Geosciences Utrecht after dilution to $3.5 \%$ salinity; nitrate and ammonia were analysed by AutoAnalyser at NIOZ (Nederlands Instituut voor Onderzoek der Zee), Texel; Mn was measured at RIVM (Rijks Instituut voor Volksgezondheid en Milieu), Bilthoven, with the use of high-resolution inductively coupled plasma mass spectrometry.

Bacterial isolation and characterization. A collection of 84 isolates was obtained by inoculating anaerobic samples of the whole interface into a wide variety of media (Supplementary Information). Isolates were tentatively identified by sequencing the $16 \mathrm{~S}$ rRNA gene, and physiological features tested included growth at different salinities and with different carbon and energy sources and terminal electron acceptors.

\section{Received 18 October; accepted 9 November 2005.}

1. De Lange, G. J., Catalano, G., Klinkhammer, G. P. \& Luther, G. W. The interface between oxic seawater and the anoxic Bannock brine; its sharpness and the consequences for the redox-related cycling of $\mathrm{Mn}$ and Ba. Mar. Chem. 31, 205-217 (1990).

2. De Lange, G. J. et al. Composition of anoxic hypersaline brines in the Tyro and Bannock basins, eastern Mediterranean. Mar. Chem. 31, 63-88 (1990).

3. Van der Wielen, P. W. J. J. et al. The enigma of prokaryotic life in deep hypersaline anoxic basins. Science 307, 121-123 (2005).

4. Benlloch, S. et al. Prokaryotic genetic diversity throughout the salinity gradient of a coastal saltern. Environ. Microbiol. 4, 349-360 (2002).

5. D'Hondt, S. et al. Distribution of microbial activities in deep subseafloor sediments. Science 306, 2216-2221 (2004).

6. Parkes, R. J. et al. Deep sub-seafloor prokaryotes stimulated at interfaces over geological time. Nature 436, 390-394 (2005).

7. Scientific Staff of Cruise Bannock 1984-12. Gypsum precipitation from cold brines in an anoxic basin in the eastern Mediterranean. Nature 314, 152-154 (1985).

8. Karner, M. B., De Long, E. F. \& Karl, D. M. Archaeal dominance in the mesopelagic zone of the Pacific Ocean. Nature 409, 507-511 (2001).

9. Henneke, E. \& De Lange, G. J. The distribution of DOC and POC in the water column and brines of the Tyro and Bannock basins. Mar. Chem. 31, 113-122 (1990).

10. LaRock, P. A., Lauer, R. D., Schwarz, J. R., Watanabe, K. K. \& Wiesenburg, D. A Microbial biomass and activity distribution in an anoxic hypersaline basin. Appl. Environ. Microbiol. 37, 466-470 (1979).

11. Luther, G. W., Catalano, G., De Lange, G. J. \& Woittiez, J. R. W. Reduced sulphur in the hypersaline anoxic basins of the Mediterranean Sea. Mar. Chem. 31, 137-152 (1990).

12. Henneke, E. \& De Lange, G. J. Sulphur speciation in anoxic hypersaline sediments from the eastern Mediterranean Sea. Geochim. Cosmochim. Acta 61, 307-321 (1997)

13. Van Cappellen, P. et al. Biogeochemical cycles of manganese and iron at the oxic-anoxic transition of a stratified marine basin (Orca Basin, Gulf of Mexico). Environ. Sci. Technol. 32, 2931-2939 (1998).

14. Eder, W., Schmidt, M., Koch, M., Garbe-Schönberg, D. \& Hubner, R. Prokaryotic phylogenetic diversity and corresponding geochemical data of the brineseawater interface of the Shaban Deep, Red Sea. Environ. Microbiol. 4, 758-763 (2002)

15. Eder, W., Ludwig, W. \& Huber, R. Novel $16 \mathrm{~S}$ rRNA gene sequences retrieved from highly saline brine sediments of Kebrit Deep, Red Sea. Arch. Microbiol. 172, 213-218 (1999).

16. Mouné, S., Caumette, P., Matheron, R. \& Willison, J. C. Molecular sequence analysis of prokaryotic diversity in the anoxic sediments underlying cyanobacterial mats of two hypersaline ponds in Mediterranean salterns. FEMS Microbiol. Ecol. 44, 117-130 (2003).

17. Hinrichs, K.-U., Hayes, J. M., Sylva, S. P., Brewer, P. G. \& DeLong, E. F. Methane-consuming archaebacteria in marine sediments. Nature 398, 802-805 (1999).

18. Canfield, D. E. \& Des Marais, D. J. Aerobic sulfate reduction in microbial mats Science 251, 1471-1473 (1991).

19. Minz, D. et al. Unexpected population distribution in a microbial mat community: sulfate-reducing bacteria localized to the highly oxic chemocline in contrast to a eukaryotic preference for anoxia. Appl. Environ. Microbiol. 65, 4659-4665 (1999)

20. Eder, W., Jahnke, L. L., Schmidt, M. \& Huber, R. Microbial diversity of the brine-seawater interface of the Kebrit Deep, Red Sea, studied via 16S rRNA 
gene sequences and cultivation methods. Appl. Environ. Microbiol. 67 3077-3085 (2001).

21. Ferrer, M. et al. Microbial enzymes mined from the Urania deep-sea hypersaline anoxic basin. Chem. Biol. 12, 895-904 (2005).

22. Coolen, M. J. L. \& Overmann, J. Functional exoenzymes as indicator of metabolically active bacteria in 124,000-year-old sapropel layers of the eastern Mediterranean Sea. Appl. Environ. Microbiol. 66, 2589-2598 (2000).

23. Coolen, M. J. L., Cypionka, H., Sass, A. M., Sass, H. \& Overmann, J. Ongoing modifications of Mediterranean Pleistocene sapropels mediated by prokaryotes. Science 296, 2407-2410 (2002).

24. Simon, M. \& Azam, F. Protein content and protein synthesis rates of planktonic marine bacteria. Mar. Ecol. Prog. Ser. 51, 201-213 (1989).

25. Takai, K. \& Horikoshi, K. Rapid detection and quantification of members of the archaeal community by quantitative PCR using fluorogenic probes. Appl. Environ. Microbiol. 66, 5066-5072 (2000).

26. Cardinale, M. et al. Comparison of different primer sets for use in automated ribosomal intergenic spacer analysis of complex bacterial communities. Appl. Environ. Microbiol. 70, 6147-6156 (2004)

27. Shannon, C. E. \& Weaver, W. The Mathematical Theory of Communication (Univ. of Illinois Press, Urbana, 1963)

28. Ludwig, W. et al. ARB: a software environment for sequence data. Nucleic Acids Res. 32, 1363-1371 (2004).
29. Pearson, T. H. \& Stanley, S. O. Comparative measurements of the redox potential of marine sediments as a rapid means of assessing the effect of organic pollution. Mar. Biol. 53, 371-379 (1979).

Supplementary Information is linked to the online version of the paper at www.nature.com/nature.

Acknowledgements We thank the master and crew of the research vessel Urania for running the cruises; R. Knoop, S. Gusic, K. Bakker and P. Krystek for the geochemical analyses; and M. Cardinale and D. B. Nedwell for discussion. K.N.T. was supported by the Fonds der Chemischen Industrie. This study was supported by the European Commission's Sustainable Marine Ecosystem program, under the BioDeep project.

Author Information The newly determined 16S rRNA sequences have been submitted to the DDBJ/NCBI/GenBank database under accession numbers AM157647-AM157656, AY547745-AY547866 and DQ289238-DQ289401. Reprints and permissions information is available at npg.nature.com/ reprintsandpermissions. The authors declare no competing financial interests Correspondence and requests for materials should be addressed to D.D. (daniele.daffonchio@unimi.it).

Biodeep Scientific Party Jean Garcin ${ }^{1}$, Boyd McKew ${ }^{2}$, Peter N. Golyshin ${ }^{3}$, Nikolaos Lampadariou ${ }^{4}$, Paraskevi Polymenakou ${ }^{4}$, Daniele Calore ${ }^{5}$, Stefano Cenedese ${ }^{5}$, Fabio Zanon ${ }^{5} \&$ Sven Hoog $^{6}$

Affiliations for participants: ${ }^{1}$ LMGEM, UMR 6117 CNRS Université de la Mediterranée, 13288 Marseille, France. ${ }^{2}$ Department of Biological Sciences, University of Essex, Colchester, Essex CO4 3SQ, UK. ${ }^{3}$ Division of Microbiology, GBF, 38122 Braunschweig, Germany. ${ }^{4}$ Institute of Marine Biology of Crete, 71003 Heraklion, Greece. ${ }^{5}$ Tecnomare SpA, ENI group, 30124 Venice, Italy. ${ }^{6}$ Technische Fachhochschule Berlin, University of Applied Science, 13353 Berlin, Germany. 